\title{
Sleep disturbances and circadian CLOCK genes in borderline personality disorder
}

\author{
Monika Fleischer • Michael Schäfer • \\ Andrew Coogan · Frank Häßler · Johannes Thome
}

Received: 16 March 2012/ Accepted: 29 June 2012

(C) Springer-Verlag 2012

\begin{abstract}
Borderline personality disorder (BPD) is characterised by a deep-reaching pattern of affective instability, incoherent identity, self-injury, suicide attempts, and disturbed interpersonal relations and lifestyle. The daily activities of BPD patients are often chaotic and disorganized, with patients often staying up late while sleeping during the day. These behavioural patterns suggest that altered circadian rhythms may be associated with BPD. Furthermore, BPD patients frequently report suffering from sleep disturbances. In this review, we overview the evidence that circadian rhythms and sleep are disturbed in BPD, and we explore the possibility that personality traits that are pertinent for BPD may be associated with circadian typology, and perhaps to circadian genotypes. With regards to sleep architecture, we review the evidence that BPD patients display altered non-REM and REM sleep. A possible cue to a deeper understanding of this temporal dysregulation might be an analysis of the circadian clock at the molecular and cellular level, as well as behavioural studies
\end{abstract}

M. Fleischer $\cdot$ M. Schäfer $\cdot$ J. Thome $(\square)$

Department of Psychiatry and Psychotherapy,

University Hospital Rostock, Gehlsheimer Straße 20,

18147 Rostock, Germany

e-mail: johannes.thome@med.uni-rostock.de

\section{A. Coogan}

Department of Psychology, National University of Ireland

Maynooth, Maynooth, Ireland

F. Häßler

Department of Child and Adolescent Psychiatry,

Psychotherapy and Neurology, University Hospital Rostock,

Rostock, Germany

J. Thome

College of Medicine, Swansea University, Swansea, UK using actigraphy and we suggest avenues for further exploration of these factors.

Keywords Actigraphy - BMAL · CRY · PER · Psychotherapy $\cdot$ Sleep disorder

\section{Introduction}

Borderline personality disorder (BPD) is characterized by a deep-reaching pattern of affective instability, incoherent identity, self-injury, suicide attempts, and disturbed interpersonal relations and lifestyle (DSM-IV). The prevalence of BPD is approximately $1-2 \%$ in the general population; BPD is more often diagnosed in women than in men and often co-occurs with Axis I disorders such as depression (96\%), anxiety disorders (64\%), posttraumatic stress disorder $(65 \%)$, substance abuse or substance dependency (64\%), ADHD (39\%), and eating disorders (53\%) (Bohus 2009). Interestingly, patients with BPD often report subjective sleep problems, especially insomnia, nightmares and dream anxiety (Bastien et al. 2008; Semiz et al. 2008). Furthermore, it has been suggested that the disturbed sleeping behaviour may alter the regulation of emotion in these patients (Harty et al. 2010), thus shaping the phenotype of the disorder.

\section{Circadian rhythms, BPD and personality}

The daily activities of BPD patients are often chaotic as well as disorganized, and the patients often stay up late while sleeping during the day (Cervena and Matousek 2005; Prasko et al. 2005), suggesting abnormal circadian timekeeping. The term circadian rhythm denotes an 
endogenously driven, circa $24-\mathrm{h}$ cycle in metabolic, endocrine and behavioural processes such as the sleepwake cycle, and circadian processes are often found to be disturbed in psychiatric and neurological conditions (Wulff et al. 2010). Verkes et al. (1996) show that BPD, along with suicide ideation and impulsiveness, was associated with diminished circadian rhythmicity in activity as assessed by actigraphy. That the diurnal variation in mood may be more pronounced in BPD patients than in those with depression and healthy controls was suggested by the study of Cowdry et al. (1991) and this observation may be in line with recent insight into how the circadian system may impact on affect (Wirz-Justice 2006; Hampp et al. 2008; Golder and Macy 2011). Analysis of the diurnal profile of salivary cortisol in female BPD patients reveals an elevated cortisol response to waking as well as a general upregulation of cortisol levels in BPD (Lieb et al. 2004). Further, the study of Wingenfeld et al. (2007) indicates that the level of nocturnal cortisol is associated with the severity of the BPD psychopathology. Given the key role for the circadian clock in determining the cortisol rhythm (Saper et al. 2005), these results may further indicate altered circadian processes in BPD. High levels of personality disorders (including BPD) have also been reported in patients suffering from circadian rhythm sleep disorders, again suggesting a link between circadian function and BPD (Dagan et al. 1996; Dagan and Eisenstein 1999). Further study is needed in order to better understand the nature of the circadian dysregulation that may accompany BPD, for example utilizing actigraphy which recently has been validated as an appropriate tool in BPD research (Huynh et al. 2010). Further, assays of molecular and endocrine circadian markers in BPD should prove useful (Baird et al. 2011).

An important factor in allowing for a deeper understanding of temporal deregulation in BPD will be analysis of circadian clock genes and their protein products, molecular factors that underpin circadian function in mammals. Clock genes contribute to the interconnection between energy metabolism, immune function, behavioural state setting and circadian rhythmicity, and may provide a direct link between the circadian and homeostatic regulation of sleep (Franken and Dijk 2009). Physiologically, circadian rhythmicity is generated by the interaction at a molecular level of endogenous clock genes such as CLOCK, BMAL1, PER1, PER2, and REV-ERB- $\alpha$ and intracellular mediators of external zeitgeber such as daylight. In mammals, the master circadian pacemaker is located in the suprachiasmatic nucleus (SCN) of the anterior hypothalamus with further semi-autonomous oscillators located throughout the brain and the periphery. Although there is evidence for abnormal circadian timekeeping and alterations of clock genes in several psychiatric populations (Wulff et al. 2010; Coogan and Thome 2011) there are-to our knowledge-no studies examining specifically polymorphisms in clock genes and/ or their expression pattern in BPD, and as noted above this is an area that warrants further exploration.

Another set of indications for associations of circadian processes with personality disorders is the associations between personality traits and diurnal preference (i.e. morningness vs. eveningness) and the possibility of a shared genetic component between these. Twin, adoption, and family studies have clearly established that personality traits have a genetic basis (Bouchard and Loehlin 2001). The five-factor model of personality differentiates the personality traits "neuroticism", "extraversion", "openness to experience", "agreeableness", and "conscientiousness", and provides the basis to interpret personality disorders as maladaptive variants of continuously distributed personality traits (Distel and Trull 2009). Clearly, "agreeableness" and "conscientiousness" are not identical. Furthermore "agreeableness" is not a trait of BPD. Terracciano et al. (2010) performed a genome-wide association scan in a genetically isolated population from Sardinia, in order to identify possible genetic variants associated with each of the five personality dimensions. Personality traits were assessed using the Revised NEO Personality Inventory (NEO-PI-R). The authors found an association between agreeableness and several single nucleotide polymorphisms (SNPs) within or close to the CLOCK gene (King et al. 1997). Although effect sizes were small (less than $1 \%$ ), the association between agreeableness and CLOCK was demonstrated in two of three replication samples (King et al. 1997).

Kendler et al. (2011) found a positive correlation between pathological BPD traits as measured by the Dimensional Assessment of Personality Pathology Basic Questionnaire and the neuroticism score of the Big Five Inventory as well as a negative correlation with the conscientiousness and agreeableness scores. Further links between circadian phenotype and personality are obtained in the study of Hogben et al. (2007) which reports that conscientiousness is a predictor of chronotype, which itself is influenced by polymorphisms in clock genes such as the variable number tandem repeat in PER3 (Ellis et al. 2009). There are numerous other studies linking circadian typology with personality traits (e.g. Caci et al. 2004; Susman et al. 2007; Adan et al. 2010). For example, and perhaps of particular reference to BPD is the recent report that using Zuckerman's Alternative five-factor personality model, a strongly biologically based paradigm, eveningness in women was associated with aggression-hostility and impulsiveness-sensation seeking (Muro et al. 2011). If chronotype is determined biologically via circadian genotype, then there may be a link from clock genes through 
chronotype onto personality features associated with BPD. Numerous studies that either have shown associations between clock gene polymorphisms and chronotype (Katzenberg et al. 1998; Archer et al. 2003; Johansson et al. 2003; Mishima et al. 2005) or have not (Barclay et al. 2011; Chang et al. 2011; Osland et al. 2011; Voinescu and Coogan 2012). Therefore, given the limited empirical data to hand at present, it remains speculative whether there is a relationship between clock genes and BPD mediated via chronotype and personality traits.

\section{Sleep disorder in patients with BPD}

Sansone et al. (2009) performed a cross-sectional study to examine the relationship between subjective sleep quality and borderline personality symptoms among 76 internal medicine outpatients. In this study, the Pittsburgh Sleep Quality Index (PSQI) was used to measure subjective sleep quality, sleep duration, sleep latency and daytime dysfunction and the Borderline Personality Scale of the Personality Diagnostic Questionnaire-4 (PDQ-4) and the Self-Harm Inventory (SHI) were used in order to evaluate borderline personality symptomatology. Significant associations were found between all PSQI subscales and the PDQ-4, and between sleep latency and the SHI. These results suggest that individuals with more severe borderline personality symptoms have more difficulties with their sleep structure than people with less pronounced borderline personality symptoms. However, the relationship between both disorders is not yet well understood.

Beyond the examination of subjective sleep quality, several research groups have used electro-encephalography (EEG) in order to obtain objective measures of sleep performance. A number of publications focus on sleep disorder, BPD and EEG (Bell et al. 1983; McNamara et al. 1984; Benson et al. 1990; Battaglia et al. 1993, 1999; De la Fuente et al. 2001, 2004; Asaad et al. 2002; Philipsen et al. 2005; Bastien et al. 2008). Bastien and colleagues (2008) looked at differences in sleep structure between BPD patients, insomnia patients (both paradoxical and psychophysiological), and healthy sleepers as control individuals. The participants of this study spent three nights in a sleep laboratory, where polysomnography recordings were conducted. The authors found that BPD subjects and insomnia subjects had longer sleep onset, shorter sleep time and lower sleep efficiency than controls. Furthermore, patients with BPD had more Non-REM stage 4 sleep than patients with paradoxical insomnia. In two other studies by Battaglia et al. (1993, 1999), BPD patients without any current co-morbid Axis I disorder were compared with healthy controls after continuous 48-h ambulatory electroencephalographic monitoring. The authors reported that REM density in subjects with BPD was significantly higher in the first REM period, and that REM latency was shorter, sleep latency and time spent awake after sleep onset were longer, while the number of awakings was higher compared to the control subjects. However, this finding was only partially replicated by Philipsen et al. (2005) who found that BPD patients without any current comorbid Axis I disorder displayed a tendency towards shortened REM latency and significantly decreased Non-REM stage 2 sleep in comparison to healthy controls. The spectral EEG analysis revealed increased delta power in total Non-REM sleep and in REM sleep in BPD subjects.

Due to the frequent co-morbidity between BPD and depression, several studies have focused on the association between BPD and depression using polysomnographic recording (Basset 2012; Huynh et al. 2012). Bell et al. (1983) studied two sub-samples of patients with major depression (with and without comorbid BPD) for a period of three nights using EEG recording. Both groups displayed a similar pattern of abnormal shortened REM latency and sleep continuity disturbances. Covarying for the Hamilton depression rating score yielded a shorter REM latency in depressive patients with BPD when compared with non-BPD depressive patients. McNamara et al. (1984) examined the sleep behaviour of ten subjects with $\mathrm{BPD}$, ten subjects with major depressive disorder (MDD) and ten healthy control subjects over two nights and reported similar findings for the BPD and MDD groups, i.e. mainly sleep continuity disturbances (lower asleep and sleep efficiency) as well as disturbances in sleep architecture (more stage 2 REM) and REM sleep (shorter REM latency; higher REM activity, density and intensity in the first REM period). Asaad et al. (2002) examined 20 patients with BPD, 20 with MDD, and 20 healthy controls. Both clinical groups differed from healthy controls with regard to longer sleep latency, lower sleep efficiency, lower stage 4 and slow wave sleep percentage, higher REM percentage, shorter REM latency, longer first REM period, and higher REM density. The MDD group differed from the BPD group with regard to longer sleep latency, lower sleep efficiency with higher arousal, shorter REM latency, higher REM density, lower first REM period, and higher REM density in the first REM period. Benson et al. (1990) examined 33 male individuals [18 with BPD with $(n=8)$ and without $(n=10)$ additional affective symptoms; 15 controls]. The whole BPD group exhibited lower waking after sleep onset, lower total sleep time, lower percent sleep and less stage 1 and stage 4 of total sleep when compared with controls. De la Fuente et al. (2001) examined 20 patients with BPD, 20 patients with MDD, and 20 healthy controls. They found that both individuals with BPD and those with MDD suffer from lower total sleep time and higher sleep onset latency, as well as higher percentage 
wakefulness when compared with controls. Furthermore, they found extended stage 2 sleep, lower stage 3 and 4 sleep, and higher REM sleep duration in BPD when compared with MDD.

Despite their methodological differences, all the mentioned studies on subjective and objective sleep measures reveal that sleep disturbances play a critical role in the pathophysiology of BPD. In summary, the sleep profile of BPD patients is significantly disturbed with regard to sleep continuity, at the same time Non-REM sleep stages as well as REM density and latency seem to be affected. Notably, the majority of studies revealed similar sleep patterns for patients with BPD and MDD, albeit less pronounced in BPD, especially with regard to shorter REM latency and higher REM density. However, the exact nature of the differences and similarities between BPD and MDD patients regarding sleep disturbances remains to be clarified. Given the literature describing circadian rhythm disturbance in MDD (Wirz-Justice 2006) and the potential for such rhythm disturbance to be a major predictor of sleep disturbance, it will be of prime importance to assess circadian function in BPD populations in parallel with the objective assessment of sleep.

\section{Therapeutic implications}

To our knowledge, no specific strategies have yet been established for the treatment of sleep disturbances in BPD. Similarly, the potential use of so-called chronotherapeutics in the treatment of BPD has not been sufficiently investigated. Plante et al. (2009) compared BPD patients' use of sedative-hypnotic medication with that of patients with other personality disorders. They found that BPD patients used a significantly higher percentage of both PRN and standing medications to help them sleep (Plante et al. 2009), demonstrating again the clinical relevance of sleep disturbances in BPD, and suggesting a high risk of drug abuse due to these disturbances. De la Fuente et al. (2002) found that carbamazepine (CBZ) increased slow wave sleep in the sleep EEG of BPD patients. CBZ was chosen because of its mood-stabilizing effect and its moderating effect on aggressive behaviour (Dalby 1975). Although CBZ did not show clinical benefits in a previous study (de la Fuente and Lotstra 1994), this finding indicates that CBZ affects objective biological sleep markers associated with sleep disturbances in BPD, although this does not imply a causal association. Interestingly, other mood stabilizers such as lithium (Yin et al. 2006) and sodium valproate (Johansson et al. 2011) have been shown to modify circadian rhythms, a factor that may be of interest in their use in BPD. Some studies have demonstrated antidepressant effects of phototherapy in subjects with non-seasonal depressive episodes. For example, Prasko et al. (2010) reported that a 6-week application of morning bright light therapy combined with administration of antidepressants could be an effective short-term augmentation strategy for BPD patients suffering from drug-resistant non-seasonal depressive episodes. This finding suggests that chronotherapeutic approaches may be useful in the management of BPD and indicate that further study to explore the use of light therapy and/or chronopharmaceutics (e.g. melatonin and agomelatine) is required.

\section{Conclusion}

There is some limited direct empirical evidence that circadian rhythms are altered in BPD, and that circadian rhythm sleep disorders are linked with BPD. Further there remains the putative link between clock genes, chronotype and personality domains that may be prescient for the understanding of novel aetioloigcal factors in BPD. Further, patients with BPD have subjective and objective sleep complaints compared to healthy controls and other clinical groups, with BPD subjects suffering from disturbed sleep physiology. The finding of disturbed EEG sleep patterns as objective measures in both BPD and MDD suggests that both conditions might share a common biological substrate. One could argue that BPD represents rather a "trait marker", whereas MDD rather a "state marker" of sleep disturbances (Steiner et al. 1988). Again, further research is needed in order to understand the causality of sleep disturbances as well as chaotic and disorganized daily activities in subjects suffering from BPD. Future studies should address themselves to examine behavioural, molecular and endocrine markers of circadian function in BPD, as well as genetic association studies for clock gene polymorphisms in BPD, and examining the links between potentially altered circadian function and impaired sleep in BPD. Finally, as noted above, there may be clinical merit in the exploration of chronobiologically based intervention strategies in symptom relief in BPD.

\section{References}

Adan A, Natale V, Caci H, Prat G (2010) Relationship between circadian typology and functional and dysfunctional impulsivity. Chronobiol Int 27:606-619

Archer SN, Robilliard DL, Skene DJ, Smits M, Williams A, Arendt J, von Schantz M (2003) A length polymorphism in the circadian clock gene Per3 is linked to delayed sleep phase syndrome and extreme diurnal preference. Sleep 26:413-415

Asaad T, Okasha T, Okasha A (2002) Sleep EEG findings in ICD-10 borderline personality disorder in Egypt. J Affect Disord $71: 11-18$ 
Baird AL, Coogan AN, Siddiqui A, Donev RM, Thome J (2011) Adult attention-deficit hyperactivity disorder is associated with alterations in circadian rhythms at the behavioural, endocrine and molecular levels. Mol Psychiatry. doi:10.1038/mp.2011.149

Barclay NL, Eley TC, Mill J, Wong CC, Zavos HM, Archer SN, Gregory AM (2011) Sleep quality and diurnal preference in a sample of young adults: associations with 5HTTLPR, PER3, and CLOCK 3111. Am J Med Genet B Neuropsychiatr Genet 156B:681-690

Basset D (2012) Borderline personality disorder and bipolar affective disorder. Spectra or spectre? A review. Aust NZ J Psychiatry 46(4):327-339

Bastien CH, Guimond S, St-Jean G, Lemelin S (2008) Signs of insomnia in borderline personality disorder individuals. J Clin Sleep Med 4(5):462-470

Battaglia M, Ferini-Strambi L, Smirne S, Bernardischi L, Bellodi L (1993) Ambulatory polysomnography of never-depressed borderline subjects: a high-risk approach to rapid eye movement latency. Biol Psychiatry 33:326-334

Battaglia M, Ferini-Strambi L, Bertella S, Bajo S, Bellodi L (1999) First-cycle rem density in never-depressed subjects with borderline personality disorder. Biol Psychiatry 45:1056-1058

Bell J, Lycaki H, Jones D, Kelwala S, Sitaram N (1983) Effect of preexisting borderline personality disorder on clinical and EEG sleep correlates of depression. Psychiatry Res 9:115-123

Benson KL, King R, Gordon D, Silva AJ, Zarcone VP Jr (1990) Sleep pattern in borderline personality disorder. JAD 18:267-273

Bohus M (2009) Borderline Persönlichkeitsstörung. In: Margraf J (ed) Lehrbuch der Verhaltenstherapie, 3rd edn. Springer, Heidelberg, pp 534-559

Bouchard TJ Jr, Loehlin JC (2001) Genes, evolution and personality. Behav Genet 31(3):243-273

Broese T, Riemann D, Hein L, Nissen C (2012) $\alpha$-Adrenergic receptor function, arousal and sleep: mechanisms and therapeutic implications. Pharmacopsychiatry (in press)

Caci H, Robert P, Boyer P (2004) Novelty seekers and impulsive subjects are low in morningness. Eur Psychiatry 19:79-84

Cervena K, Matousek M (2005) Sleep disturbance in patients treated for panic disorder. Sleep Med 6(2):149-153

Chang AM, Buch AM, Bradstreet DS, Klements DJ, Duffy JF (2011) Human diurnal preference and circadian rhythmicity are not associated with the CLOCK $3111 \mathrm{C} / \mathrm{T}$ gene polymorphism. J Biol Rhythms 26:276-279

Coogan AN, Thome J (2011) Chronotherapeutics and psychiatry: setting the clock to relieve the symptoms. World J Biol Psychiatry 12(Suppl 1):40-43

Cowdry RW, Gardner DL, O'Leary KM, Leibenluft E, Rubinow DR (1991) Mood variability: a study of four groups. Am J Psychiatry 148:1505-1511

Dagan Y, Eisenstein M (1999) Circadian rhythm sleep disorders: toward a more precise definition and diagnosis. Chronobiol Int $16: 213-222$

Dagan Y, Sela H, Omer H, Hallis D, Dar R (1996) High prevalence of personality disorders among circadian rhythm sleep disorders (CRSD) patients. J Psychosom Res 41:357-363

Dalby AM (1975) Behavioral effects of carbamazepine. Adv Neurol $11: 331-344$

De la Fuente JM, Lotstra F (1994) A trial of carbamazepine in borderline personality disorder. Eur Neuropsychopharmacol 4(4):479-486

De la Fuente JM, Bobes J, Vizuete C, Mendlewicz J (2001) SleepEEG in borderline patients without concomitant major depression: a comparison with major depressives an normal control subjects. Psychiatry Res 105:87-95

De la Fuente JM, Bobes J, Vizuete C, Mendlewicz J (2002) Effects of carbamazepine on dexamethasone suppression and sleep electroencephalography in borderline personality disorder. Neuropsychobiology 45(3):113-119

De la Fuente JM, Bobes J, Morlan I, Bascaran MT, Vizuete C, Linkowski $\mathrm{P}$ et al (2004) Is the biological nature of depressive symptoms in borderline patients without concomitant Axis I pathology idiosyncratic? Sleep EEG comparison with recurrent brief, major depression and control subjects. Psychiatry Res 129:65-73

Distel MA, Trull TJ (2009) The five-factor model of personality and borderline personality disorder: a genetic analysis of comorbidity. Biol Psychiatry 66(12):1131-1138

Ellis J, von Schantz M, Jones KH, Archer SN (2009) Association between specific diurnal preference questionnaire items and PER3 VNTR genotype. Chronobiol Int 26(3):464-473

Franken P, Dijk DJ (2009) Circadian clock genes and sleep homeostasis. Eur J Neurosci 29:1820-1829

Golder SA, Macy MW (2011) Diurnal and seasonal mood vary with work, sleep, and daylength across diverse cultures. Science 333:1878-1881

Hampp G, Ripperger JA, Houben T, Schmutz I, Blex C, Perreau-Lenz S, Brunk I, Spanagel R, Ahnert-Hilger G, Meijer JH, Albrecht U (2008) Regulation of monoamine oxidase A by circadian-clock components implies clock influence on mood. Curr Biol 18:678-683

Harty L, Duckworth R, Thompson A, Stuewig J, Tangney JP (2010) Are inmates' subjective sleep problems associated with borderline personality, psychopathy, and antisocial personality independent of depression and substance dependence? J Forens Psychiatry Psychol 21(1):23-39

Hogben AL, Ellis J, Archer SN, von Schantz M (2007) Conscientiousness is a predictor of diurnal preference. Chronobiol Int 24(6):1249-1254

Huynh C, Guilé JM, Breton JJ, Desrosiers L, Cohen D, Godbout R (2010) Is it possible to study sleep-wake patterns in adolescent borderline personality disorder? An actigraphic feasibility study. Int J Adolesc Med Health 22:547-560

Huynh C, Guilé JM, Godbout R (2012) Polysomnographic studies on sleep in adult borderline personality disorder. Presse Med 41(2):e63-e75

Johansson C, Willeit M, Smedh C, Ekholm J, Paunio T, Kieseppä T, Lichtermann D, Praschak-Rieder N, Neumeister A, Nilsson LG, Kasper S, Peltonen L, Adolfsson R, Schalling M, Partonen T (2003) Circadian clock-related polymorphisms in seasonal affective disorder and their relevance to diurnal preference. Neuropsychopharmacology 28:734-739

Johansson AS, Brask J, Owe-Larsson B, Hetta J, Lundkvist GB (2011) Valproic acid phase shifts the rhythmic expression of Period2:luciferase. J Biol Rhythms 26:541-551

Katzenberg D, Young T, Finn L, Lin L, King DP, Takahashi JS, Mignot E (1998) A clock polymorphism associated with human diurnal preference. Sleep 21:569-576

Kendler KS, Myers J, Reichborn-Kjennerud T (2011) Borderline personality disorder traits and their relationship with dimension of normative personality: a web-based cohort and twin study. Acta Psychiatr Scand 123(5):349-359

King DP, Zhao Y, Sangoram AM, Wilsbacher LD, Tanaka M, Antoch MP et al (1997) Positional cloning of the mouse circadian clock gene. Cell 89(4):641-653

Lieb K, Rexhausen JE, Kahl KG, Schweiger U, Philipsen A, Hellhammer DH, Bohus M (2004) Increased diurnal salivary cortisol in women with borderline personality disorder. J Psychiatr Res 38:559-565

McNamara E, Reynolds CF III, Soloff PH, Mathias R, Rossi A, Spiker D (1984) EEG sleep evaluation of depression in borderline patients. Am J Psychiatry 141(2):182-186

Mishima K, Tozawa T, Satoh K, Saitoh H, Mishima Y (2005) The $3111 \mathrm{~T} / \mathrm{C}$ polymorphism of hClock is associated with evening 
preference and delayed sleep timing in a Japanese population sample. Am J Med Genet B Neuropsychiatr Genet 133B: 101-104

Muro A, Gomà-i-Freixanet M, Adan A, Cladellas R (2011) Circadian typology, age, and the alternative five-factor personality model in an adult women sample. Chronobiol Int 28:690-696

Osland TM, Bjorvatn BR, Steen VM, Pallesen S1 (2011) Association study of a variable-number tandem repeat polymorphism in the clock gene PERIOD3 and chronotype in Norwegian university students. Chronobiol Int 28:764-770

Philipsen A, Feige B, Al-Shajlawi A, Schmahl Ch, Bohus M, Richter $\mathrm{H}$ et al (2005) Increased delta power and discrepancies in objective and subjective sleep measurement in borderline personality disorder. J Psychiatr Res 39:489-498

Plante DT, Zanarini MC, Frankenburg FR, Fitzmaurice GM (2009) Sedative-hypnotic use in patents with borderline personality disorder and axis II comparison subjects. J Pers Disord 23(6):563-571

Prasko J, Houbova P, Novak, Zalesky R, Espa-Cervena K, Paskova B (2005) Influence of personality disorder on the treatment of panic disorder-comparison study. Neuro Endocrinol Lett 26(6):667-674

Prasko J, Brunovsky M, Latalova K, Grambal A, Raszka M, Vyskocilova J et al (2010) Augmentation of antidepressants with bright light therapy in patients with comorbid depression and borderline personality disorder. Biomed Pap Med Fac Univ Palacky Olomouc Czech Repub 154(4):355-361

Sansone RA, Edwards HC, Forbis JS (2009) Sleep quality in borderline personality disorder: a cross-sectional study. Prim Care Copanion J Clin Psychiatry 12(5). doi:10.4088/PCC. 09m00919

Saper CB, Lu J, Chou TC, Gooley J (2005) The hypothalamic integrator for circadian rhythms. Trends Neurosci 28:152-157

Semiz UB, Basoglu C, Ebrinc S, Cetin M (2008) Nightmare disorder, dream anxiety, and subjective sleep quality in patients with borderline personality disorder. Psychiatry Clin Neurosci $62: 48-55$

Steiner M, Links PS, Korzekwa M (1988) Biological markers in borderline personality disorder: an overview. Can J Psychiatry 33:350-354

Susman EJ, Dockray S, Schiefelbein VL, Herwehe S, Heaton JA, Dorn LD (2007) Morningness/eveningness, morning-to-afternoon cortisol ratio, and antisocial behavior problems during puberty. Dev Psychol 43:811-822

Terracciano A, Sanna S, Uda M, Deiana B, Usala G, Busonero F et al (2010) Genome-wide association scan for five major dimensions of personality. Mol Psychiatry 15(6):647-656

Verkes RJ, Kerkhof GA, Beld E, Hengeveld MW, van Kempen GM (1996) Suicidality, circadian activity rhythms and platelet serotonergic measures in patients with recurrent suicidal behaviour. Acta Psychiatr Scand 93:27-34

Voinescu BI, Coogan AN (2012) A variable number tandem repeat polymorphism in PER3 is not associated with chronotype in a population with self-reported sleep problems. Sleep Biol Rhythm 10:23-26

Wingenfeld K, Driessen M, Adam B, Hill A (2007) Overnight urinary cortisol release in women with borderline personality disorder depends on comorbid PTSD and depressive psychopathology. Eur Psychiatry 22:309-312

Wirz-Justice A (2006) Biological rhythm disturbances in mood disorders. Int Clin Psychopharmacol 21(Suppl 1):S11-S15

Wulff K, Gatti S, Wettstein JG, Foster RG (2010) Sleep and circadian rhythm disruption in psychiatric and neurodegenerative disease. Nat Rev Neurosci 11:589-599

DSM-IV Diagnostic and Statistical Manual of Mental Disorders, 4th edn. American Psychiatric Association, Washington DC

Yin L, Wang J, Klein PS, Lazar MA (2006) Nuclear receptor Reverbalpha is a critical lithium-sensitive component of the circadian clock. Science 311:1002-1005 